\title{
Kennzeichnung von Wildgulasch und Fleischerzeugnissen aus Wild
}

\author{
Dr. G. Schulze \\ Bayerisches Landesamt für Gesundheit und Lebensmittelsicherheit, Erlangen
}

Ein Hersteller produziert Wildgulasch, das zu etwa einem Drittel aus Hirsch- und zu zwei Dritteln aus Kängurufleisch besteht. Das Erzeugnis soll roh als Fertigpackung in Verkehr gebracht werden. Hinsichtlich der Kennzeichnung eines solchen Produktes gibt es unterschiedliche Sachverständigenmeinungen:

1. Kennzeichnungsvorschlag: Verkehrsbezeichnung „gemischter Wildgulasch“ mit Auflistung der verwendeten Tierarten in der Zutatenliste ohne Mengenangabe, mit folgender Argumentation: Nach allgemeiner Verkehrsauffassung (Leitsätze für Fleisch und Fleischerzeugnisse Ziffer: 2.511.3) besteht Gulasch aus gestückeltem Rindfleisch. Bei ausschließlicher oder teilweiser Verwendung von Fleisch anderer Tierarten ist dies in unmittelbarer Wortverbindung mit der Bezeichnung „Gulasch“ anzugeben. Beispielhaft sind hier aufgeführt: „Kalbsgulasch“, „gemischter Gulasch“.

Bezogen auf das Erzeugnis „Wildgulasch“ heißt dies: Bei ausschließlicher Verwendung einer anderen Tierart muss diese in Verbindung mit der Verkehrsbezeichnung genannt werden. Werden mehrere Tierarten verwendet, so reicht die Angabe „gemischter Gulasch“ aus. Die Angabe der verwendeten Tierarten erfolgt dann im Zutatenverzeichnis, eine Mengenkennzeichnung nach § 8 LMKV ist nicht erforderlich.

Der Begriff „Wild“ wird als Sammelbegriff für alle möglichen wild lebenden Tierarten verwendet und schließt daher ist auch das Känguru mit ein.

2. Kennzeichnungsvorschlag: Verkehrsbezeichnung „Kängurugulasch mit Hirsch“, mit folgender Argumentation: Die Bezeichnung „gemischter Wildgulasch“ für ein Erzeugnis, das überwiegend aus Kängurufleisch besteht, ist unzutreffend, da Känguru nach allgemeiner Verkehrsauffassung nicht unter den Begriff „Wild“ fällt.

In Anhang I der VO (EG) 853/2004 unter Nr.1.5. „frei lebendes Wild“ als frei lebende Huf- und Hasentiere sowie andere Landsäugetiere, die nach dem geltenden Recht des betreffenden
Mitgliedstaates als Wild gelten, definiert. Nach dem Bundesjagdgesetz §2 Abs. 1 fallen sowohl Haar- als auch Federwild unter das Jagdrecht. Zum Haarwild gehören u. a. Elchwild, Rotwild, Damwild, Rehwild, Sikawild, Gamswild Wildkaninchen, Feldhase etc.. Nicht aufgelistet wird Känguru oder andere exotische Wildarten wie beispielsweise Antilopen, Springbock u.ä.. Demnach fällt Känguru nicht unter den Begriff „Wild“ und muss als Tierart in der Verkehrsbezeichnung genannt werden. Darüber hinaus ist eine Mengenangabe des verwendeten Kängurufleisches in der Zutatenliste erforderlich, wenn dies in der Verkehrsbezeichnung genannt ist.

\section{In diesem Zusammenhang stellen sich weitere Fragen:}

a. Gibt es Konsequenzen für die Kennzeichnung, wenn ein Teil des Ausgangsmaterials von Gehegewild stammt? Unserer Meinung nach fällt Gehegewild nach Anhang I der VO (EG) 853/2004 unter Nr. 1.5. „frei lebendes Wild“, wenn es in einem geschlossenen Gehege unter ähnlichen Bedingungen wie frei lebendes Wild lebt.

b. Kann ein Fleischerzeugnis aus „Wildfleisch“ analog zu dem Begriff „Geflügelfleisch“ beispielsweise als „Wildfleischwurst“ bezeichnet werden oder muss die Tierart in Zusammenhang mit der Verkehrsbezeichnung angegeben werden?

Wenn es sich um Wild i.S. der Begriffsbestimmung in Anhang I der VO (EG) 853/2004 unter Nr. 1.5. „frei lebendes Wild“ handelt ist unseres Erachtens eine Verkehrsbezeichnung „Wildfleischwurst“ o. Ä. möglich.

\section{Beschluss (einstimmig)}

Unter dem Begriff „Wild“ wird üblicherweise das zum Verzehr bestimmte, jagdbare europäische Haar- und Federwild verstanden. 